\title{
Un estudio exploratorio sobre el uso de las tecnologías de la información y comunicación en adolescentes
}

\author{
SANDRA ROJAS RODRÍGUEZ \\ al189941@uji.es \\ ENCARNA MARTÍN-LORENTE \\ encarna.martinlorente@gmail.com \\ JUANA BRETÓN-LÓPEZ \\ breton@uji.es
}

\section{Resumen}

Introducción: Las tecnologías de la información y la comunicación (TIC) han supuesto grandes beneficios. Sin embargo, pueden ocasionar graves problemas derivados del uso excesivo de las mismas. Una población de riesgo son los adolescentes, debido a las características que los definen y a que son quienes más las utilizan. El objetivo de esta investigación es estudiar el uso que hacen los adolescentes de un conjunto de tecnologías y comprobar si existe un abuso de las TIC en esta población. Método: Los participantes eran 47 estudiantes de un instituto de educación secundaria de la provincia de Castellón. Se les administró el cuestionario de detección de nuevas adicciones (DENA) de Labrador, Becoña y Villadangos (2008). Resultados: Los resultados hallados fueron un uso extendido de internet, teléfono móvil y televisión, pues un $91,5 \%$, un $89,1 \%$ y un $76,1 \%$ respectivamente utilizaban estas tecnologías a diario. Además, se encontró un uso elevado de internet y del teléfono móvil, pues un $46,7 \%$ y un $65 \%$ respectivamente los utilizaba de 3 a 6 horas diarias. También se hallaron importantes problemas derivados del uso de internet y del teléfono móvil, pues un $6,4 \%$ y un $8,5 \%$ respectivamente indicaron tener siempre problemas por su uso. Así mismo, se hallaron correlaciones significativas entre la frecuencia de uso y la frecuencia de problemas percibidos de internet $(r=0,296 ; p=0,043)$, videojuegos $(r=0,588 ; p=0,000)$ y televisión ( $r=0,355 ; p=0,017)$. Conclusiones: El estudio revela la necesidad de difundir pautas para un uso saludable de las TIC. Así mismo, es importante hacer énfasis en la necesidad de seguir estudiando esta problemática.

Palabras clave: tecnología, abuso, adolescentes, internet, teléfono móvil.

\section{Abstract}

Introduction: Information and Communication Technologies (ICT) have brought great benefits. However, they can also cause serious problems because of their excessive use. Teenagers are considered a population at risk due to their characteristics and because they are the ones who most use ICTS. The objective of this research is to 
analyse the use of a set of technologies by adolescents and to find out if there is an abuse of ICTs in this population. Method: Participants were 47 students of one specific high school in the province of Castellon. The detection of new addictions questionnaire (DENA) by Labrador, Becoña and Villadangos (2008) was administered. Results: Results showed an extended use of the Internet, mobile phone and television among teenagers, as $91.5 \%, 89.1 \%$ and $76.1 \%$ respectively use these technologies daily. In addition, a $46.7 \%$ for the Internet and $65 \%$ for mobile phone use from 3 to 6 hours per day. Moreover, a high percentage of problems resulting from the use of the Internet and mobile phone were found, as $6.4 \%$ and $8.5 \%$ respectively have always perceived problems of use. Also, significant correlations between frequency of use and frequency of perceived problems were found on the Internet $(p=0.043 ; r=0.296)$, video games $(r=0.588 ; p=0.000)$ and television $(r=0.355 ; p=0.017)$. Conclusions: The study shows that it is necessary to disseminate guidelines for a healthy use of ICTS and to continue studying this problem.

Keywords: technology, abuse, addiction, adolescents, Internet, mobile phone.

\section{Introducción}

Las tecnologías de la información y la comunicación (TIC) son herramientas que proporcionan grandes beneficios, como la obtención y transmisión de manera rápida de información, la comunicación entre personas de forma instantánea y el entretenimiento, entre otros (Labrador y Villadangos, 2009). Sin embargo, el inconveniente que presentan es cuando el uso de las mismas se convierte en abusivo o incluso adictivo, por lo que pasan a ser una parte central de la vida de la persona, ya que se les dedica gran cantidad de tiempo y atención y se dejan de realizar otras actividades, lo que genera una grave interferencia en la vida de las personas (Labrador y Villadangos, 2009).

No obstante, no existe un acuerdo claro sobre qué criterios caracterizan la adicción a las TIC. En algunos casos se considera la frecuencia o duración del uso de las mismas; sin embargo, la cantidad de horas dedicadas a una actividad no parece ser suficiente para establecer un problema adictivo (Labrador y Villadangos, 2010). Asimismo, ni en el anterior Manual Diagnóstico y Estadístico de los Trastornos Mentales (DSM-IV) ni en el actual (DSM-V), existen criterios establecidos para determinar si una persona presenta adicción a las TIC, ni tampoco en la clasificación internacional de las enfermedades (CIE-10). Sin embargo, los criterios se pueden inferir a partir de las características de las adicciones comportamentales. De esta manera, como señala Griffiths (2005), los componentes comunes en la adicción, ya sea comportamental o tóxica, son: saliencia, cambio de humor, tolerancia, síndrome de abstinencia, conflicto y recaída. Por tanto, una conducta será adictiva si es muy importante para la persona y domina sus pensamientos, sentimientos y comportamientos (esto es, saliencia). Así mismo, provocará un cambio en el humor, que será resultado de la implicación de la persona en una determinada actividad adictiva. También ocasionará tolerancia, que es el proceso en el que se requiere aumentar la cantidad de una determinada conducta para alcanzar los mismos efectos que esta producía al comienzo. De la misma manera, producirá síndrome de abstinencia en la persona; este es entendido como estados emocionales o efectos físicos displacenteros que ocurren cuando se deja de realizar la actividad. Además, provocará distintos tipos de conflictos, que pueden ser: entre el adicto y aquellos que le rodean (conflicto interpersonal), en el mismo in- 
dividuo (conflicto intrapsíquico) o conflicto de la actividad con otras actividades, como pueden ser el trabajo, los estudios, la vida social, etc. Finalmente, se producirán recaídas, que son la tendencia a volver a realizar la conducta problemática incluso tras lograr permanecer un largo tiempo de abstinencia y control.

Además, cabe tener en cuenta que en el DSM-V (APA, 2013) se proponen para estudiar en el futuro los criterios diagnósticos del trastorno de juego en internet. Sin embargo, se requieren más investigaciones para establecerlos. Estos criterios estarían relacionados con el uso persistente y recurrente de internet para participar en juegos, que conlleva un deterioro o malestar clínicamente significativo y que se manifiesta durante un periodo de 12 meses. Por tanto, estarían relacionados con la adicción a los juegos de internet, por lo que debido a su semejanza con la adicción a las TIC, podrían ser adaptados y utilizarse para diagnosticar la adicción a las distintas tecnologías.

Los perjuicios que pueden causar estas adicciones se pueden dar a cualquier edad, aunque son los adolescentes quienes constituyen un grupo de mayor riesgo. Esto es debido a que la adolescencia es un periodo de mayor vulnerabilidad, por ser una edad en la que se está produciendo el desarrollo del control cognitivo (Casey, Tottenham, Liston y Durston, 2005) y el desarrollo de las habilidades de ajuste (Liu y Potenza, 2007). Así mismo, son los adolescentes quienes tienen mayor tendencia a buscar sensaciones nuevas, quienes más utilizan estas tecnologías y están más familiarizados con ellas (Sánchez-Carbonell, Beranuy, CasteIlana, Chamarro y Oberst, 2008). Además, las utilizan para jugar, comunicarse con otras personas y como fuente de conocimiento en su proceso de aprendizaje (Martín, Beltrán y Pérez, 2003), lo que lleva a convertir a las TIC en importantes e imprescindibles en sus vidas.

Algunos datos previos relacionados con el porcentaje de uso, abuso y adicción de estas tecnologías en los adolescentes son los siguientes:

En cuanto a internet, según el estudio realizado por la Fundación Pfizer (2009), el 98 \% de los jóvenes españoles de 11 a 20 años era usuario del mismo; de ese porcentaje, siete de cada diez afirmaron acceder a la red por un tiempo diario de, al menos, 1,5 horas, pero en torno al $3 \%$ o al $6 \%$ de los mismos hacía un uso abusivo de internet (Echeburúa y Corral, 2010). Estos datos se ven confirmados en el estudio realizado por Carbonell y cols. (2012), pues un $6,1 \%$ de los participantes mostraba problemas frecuentes del uso de internet.

En cuanto a los datos relacionados con el uso abusivo del móvil, en el trabajo realizado por Chóliz, Villanueva y Chóliz (2009) se halló que el $95 \%$ de los adolescentes del estudio tenían móvil, cifra que llegaba al $99 \%$ en adolescentes de 17 y 18 años. El 62,9\% de los menores lo tenían siempre conectado, incluso por la noche. En otro estudio posterior realizado por Carbonell y cols. (2012) se comprobó que un 2,8 \% de los participantes presentaba problemas frecuentes con el uso del teléfono móvil.

En cuanto a los videojuegos, en el estudio de Protégeles de 2005 para el Defensor del Menor, se comprobó que el $69 \%$ de los menores jugaba habitualmente con los mismos, pero esta prevalencia iba disminuyendo con la edad. También, los menores reconocieron que los videojuegos les quitaban tiempo de realizar otras actividades; así pues, el $28 \%$ reconoció que le quitaba tiempo de estudio, el $21 \%$ de estar con la familia y el $15 \%$ de estar con los amigos. Finalmente, el $14 \%$ reconoció tener conductas problemáticas.

En otro estudio, Labrador y Villadangos (2010) administraron a una población de adolescentes el cuestionario de detección de nuevas adicciones (DENA), cuyos resultados indicaron que la tecnología más utilizada era la televisión, pues un 97,23 \% reconoció utilizarla con frecuencia o siempre, seguida de internet con un $87,43 \%$ y teléfono móvil con un $80,81 \%$, siendo los videojuegos los menos utilizados, solamente por un $55,26 \%$. También se encontró una correlación positiva entre el tiempo de uso de las distintas tecnologías y la percepción de problemas; por tanto, cuanto más utilizan las TIC, más creen que pueden causarles problemas. 
Así pues, la televisión es considerada como la que más problemas les genera, seguida de internet y teléfono móvil, mientras que son los videojuegos los considerados como menos problemáticos.

Con todo lo anterior, teniendo en cuenta las graves consecuencias que puede tener el uso adictivo de estas tecnologias y que son los adolescentes los que constituyen el grupo de mayor riesgo, el objetivo de la presente investigación es estudiar el uso que hacen los adolescentes de un conjunto de tecnologías, clasificar estas tecnologías de acuerdo a su frecuencia de uso y poner en relación la frecuencia de uso con la existencia de problemas en los adolescentes. Todo ello con la finalidad de conocer el uso que hacen de las mismas y concienciarles acerca de esta problemática, así como proporcionar a sus padres las pautas de prevención adecuadas.

\section{Método}

\section{Participantes}

En el estudio participaron una muestra de 47 estudiantes de educación secundaria de un centro educativo de la provincia de Castellón. El 53,2 \% fueron mujeres y el 46,8 \% varones. La edad de los participantes comprendía de los 16 a los 18 años, con una edad media de 16,51 (DT $=0,621)$.

\section{Medidas}

El instrumento utilizado fue el cuestionario de detección de nuevas adicciones (DENA) (Labrador y Villadangos, 2010). Las variables que se tuvieron en cuenta para realizar el presente estudio fueron la edad, el sexo, los datos relacionados con la frecuencia de uso de las tecnologías (internet, teléfono móvil, videojuegos y televisión), la percepción subjetiva de problemas derivados de su uso y el tiempo dedicado a ellas al día.

\section{Diseño}

Se realizó un estudio descriptivo mediante observación, de acuerdo a la clasificación establecida por Montero y León (2005).

\section{Procedimiento}

Los datos se recogieron durante el curso académico 2013-2014. El cuestionario fue administrado por una psicóloga en el centro escolar. Las autoridades académicas del centro dieron la aprobación a la administración del cuestionario y hubo el correspondiente permiso de los padres. La aplicación fue colectiva, aunque cada estudiante respondía de manera individual y anónima a su cuestionario. Se recogieron los datos y se trasladaron a la base de datos para su posterior análisis. Se acordó con el centro informar debidamente de los resultados obtenidos y planificar una charla con los padres, tanto para mostrarles los resultados obtenidos como para enseñarles pautas de prevención sobre este tipo de abuso o adicción comportamental. 


\section{Resultados}

En cuanto a la frecuencia de uso de las tecnologías, los participantes reconocieron que las tecnologías que utilizan con mayor frecuencia son internet, teléfono móvil y televisión. Así pues, la mayoría de los adolescentes hacen un uso diario de internet (91,5\% de los mismos), teléfono móvil $(89,1 \%)$ y televisión $(76,1 \%)$, así como otros las utilizan alguna vez por semana, en el caso de internet $(6,4 \%)$, del teléfono móvil $(8,7 \%)$ y de la televisión $(23,9 \%)$. Por otro lado, el uso de los videojuegos es menor si se compara con el uso que hacen de las otras tecnologías (tabla 1).

Tabla 1

Frecuencia de uso de las tecnologías

\begin{tabular}{lcccccccc}
\hline & \multicolumn{2}{c}{ Nunca } & \multicolumn{2}{c}{ Alguna vez al mes } & \multicolumn{2}{c}{$\begin{array}{c}\text { Alguna vez } \\
\text { a la semana }\end{array}$} & \multicolumn{2}{c}{ Todos los días } \\
\hline & $\mathrm{N}$ & $\%$ & $\mathrm{~N}$ & $\%$ & $\mathrm{~N}$ & $\%$ & $\mathrm{~N}$ & $\%$ \\
\hline Internet & - & - & 1 & 2,1 & 3 & 6,4 & 43 & 91,5 \\
Videojuegos & 21 & 44,7 & 13 & 27,7 & 10 & 21,3 & 3 & 6,4 \\
Teléfono móvil & 1 & 2,2 & - & - & 4 & 8,7 & 41 & 89,1 \\
Televisión & - & - & - & - & 11 & 23,9 & 35 & 76,1 \\
\hline
\end{tabular}

En cuanto al tiempo dedicado a las TIC al día (en horas), existe un porcentaje importante de los adolescentes que utilizan internet y el teléfono móvil una gran cantidad de horas al día. De esta manera, en cuanto al uso de internet, un 46,7 \% lo utiliza de 3 a 6 horas al día y, respecto al uso del teléfono móvil, un $65 \%$ lo utiliza de 3 a 6 horas diarias. No obstante, respecto a la televisión y los videojuegos, los adolescentes hacen un menor uso al día. Así pues, en cuanto a la televisión, un 50,0 \% la ven 1 hora al día, mientras que un 31,8 \% la ven 2 horas al día y un $11,4 \%$ la ven 3 horas al día. En relación a los videojuegos, las horas que les dedican al día aquellos que los utilizan oscilan entre 1 y 3 horas, siendo mayor el porcentaje que los utiliza 1 hora al día (68,8 \%), seguido por el que los utiliza 2 horas al día $(25,0 \%)$ (tabla 2$)$.

Tabla 2

Tiempo dedicado a las TIC al día (horas)

\begin{tabular}{lccccccccccccc}
\hline & \multicolumn{1}{c}{1} & \multicolumn{2}{c}{2} & & & & & 4 & & 5 & \multicolumn{2}{c}{6} \\
\hline & $\mathrm{N}$ & $\%$ & $\mathrm{~N}$ & $\%$ & $\mathrm{~N}$ & $\%$ & $\mathrm{~N}$ & $\%$ & $\mathrm{~N}$ & $\%$ & $\mathrm{~N}$ & $\%$ \\
\hline Internet & 16 & 35,6 & 8 & 17,8 & 8 & 17,8 & 7 & 15,6 & 1 & 2,2 & 5 & 11,1 \\
Videojuegos & 11 & 68,8 & 4 & 25,0 & 1 & 6,3 & - & - & - & - & - & - \\
Telf. móvil & 10 & 25,0 & 4 & 10,0 & 4 & 10,0 & 9 & 22,5 & 5 & 12,5 & 8 & 20,0 \\
Televisión & 22 & 50,0 & 14 & 31,8 & 5 & 11,4 & 2 & 4,5 & - & - & 1 & 2,3 \\
\hline
\end{tabular}

*Resultados obtenidos en población válida. 
En lo que se refiere a la frecuencia de problemas percibidos debido al uso de las tecnologías, los participantes consideran que internet y el teléfono móvil son las tecnologías que mayores problemas les generan. En concreto, perciben que internet les genera problemas con frecuencia a un $8,5 \%$ de los adolescentes y siempre a un $6,4 \%$, y el teléfono móvil les genera problemas con frecuencia a un $10,6 \%$ y siempre a un $8,5 \%$. En cuanto a la televisión, parece generar problemas con frecuencia a un $8,7 \%$ y siempre a un $2,2 \%$. Por lo que respecta a los videojuegos, ninguno de los adolescentes contestó que le generen problemas con frecuencia y solo un 2,1 \% contestó que les genera problemas siempre (tabla 3 ).

Tabla 3

Frecuencia de problemas percibidos debido al uso de las tecnologías

\begin{tabular}{lcccccccc}
\hline & \multicolumn{2}{c}{ Nunca } & \multicolumn{2}{c}{ A veces } & \multicolumn{2}{c}{ Con frecuencia } & \multicolumn{2}{c}{ Siempre } \\
\hline & $\mathrm{N}$ & $\%$ & $\mathrm{~N}$ & $\%$ & $\mathrm{~N}$ & $\%$ & $\mathrm{~N}$ & $\%$ \\
\hline Internet & 17 & 36,2 & 23 & 48,9 & 4 & 8,5 & 3 & 6,4 \\
Videojuegos & 40 & 85,1 & 6 & 12,8 & - & - & 1 & 2,1 \\
Teléfono móvil & 16 & 34,0 & 22 & 46,8 & 5 & 10,6 & 4 & 8,5 \\
Televisión & 30 & 65,2 & 11 & 23,9 & 4 & 8,7 & 1 & 2,2 \\
\hline
\end{tabular}

En referencia a la relación entre la frecuencia de uso de las TIC y los problemas percibidos por los adolescentes, se han encontrado relaciones significativas en el caso de internet $(r=0,296 ; p=0,043)$, videojuegos $(r=0,588 ; p=0,000)$ y televisión $(r=0,355 ; p=0,017)$, sin embargo no se han encontrado en el caso del teléfono móvil (tabla 4).

Tabla 4

$r$ de Pearson entre la frecuencia de uso de TIC y los problemas percibidos

\begin{tabular}{|c|c|c|c|c|c|}
\hline & & M & D.T. & $r$ & $P$ \\
\hline \multirow{2}{*}{ Internet } & Frecuencia & 3,89 & 0,375 & \multirow{2}{*}{0,296} & \multirow{2}{*}{0,043} \\
\hline & Problemas & 1,85 & 0,834 & & \\
\hline \multirow{2}{*}{ Videojuegos } & Frecuencia & 1,89 & 0,961 & \multirow{2}{*}{0,588} & \multirow{2}{*}{0,000} \\
\hline & Problemas & 1,19 & 0,537 & & \\
\hline \multirow{2}{*}{ Teléfono móvil } & Frecuencia & 3,85 & 0,515 & \multirow{2}{*}{0,275} & \multirow{2}{*}{0,064} \\
\hline & Problemas & 1,94 & 0,895 & & \\
\hline \multirow{2}{*}{ Televisión } & Frecuencia & 3,76 & 0,431 & \multirow{2}{*}{0,355} & \multirow{2}{*}{0,017} \\
\hline & Problemas & 1,48 & 0,752 & & \\
\hline
\end{tabular}




\section{Discusión y conclusiones}

Los resultados obtenidos en este estudio muestran que las tecnologías más utilizadas por los adolescentes son internet, teléfono móvil y televisión. Estos datos van en la línea de estudios anteriores sobre el uso de las distintas tecnologías, ya que en el estudio realizado por la Fundación Pfizer (2009) el 98 \% de los jóvenes españoles era usuario de internet (siendo $97,9 \%$ en nuestro estudio los adolescentes que lo utilizan diariamente o alguna vez a la semana). Lo mismo ocurre en el caso del teléfono móvil, incluso puede percibirse un aumento del uso del mismo en comparación con estudios anteriores pues, como Chóliz y cols. (2009) encontraron, el $62,9 \%$ de los adolescentes lo tenían siempre conectado, siendo un $97,8 \%$ en nuestro estudio los adolescentes que hacen un uso del mismo a diario o alguna vez a la semana. De la misma manera, en cuanto a los videojuegos, el porcentaje de uso disminuye comparándolo con las otras tecnologías, pues como indica el estudio de Protégeles del 2005, el $69 \%$ de los menores jugaba habitualmente con videojuegos, siendo este uso todavía menor por parte de los adolescentes en nuestro estudio $(27,7 \%$ indicaron utilizarlo a diario o alguna vez a la semana).

Así mismo, los resultados obtenidos en el presente estudio son similares a los encontrados en el estudio realizado por Labrador y Villadangos (2010), quienes también utilizaron el cuestionario DENA y hallaron que la tecnología más utilizada por los adolescentes era la televisión con un uso de $97,23 \%$ de los mismos con frecuencia o siempre (100\% en nuestro estudio), seguida de internet con un $87,43 \%$ de los mismos (97,9\% en nuestro estudio) y teléfono móvil $80,81 \%$ (97,8 \% en nuestro estudio), siendo la menos utilizada los videojuegos por un $55,26 \%$ (27,7 \% en nuestro estudio). Estos datos reflejan que parece haber un aumento del uso de internet y del teléfono móvil en los adolescentes de nuestro estudio, mientras que en el caso de la televisión el uso se mantiene similar y disminuye en el caso de los videojuegos.

En el caso de las horas que los adolescentes utilizan las tecnologías al día, se encontró una gran proporción de los mismos que dedicaban entre 3 y 6 horas al día tanto a internet como al teléfono móvil. Cabe tener en cuenta que, en la mayoría de los casos, este uso no es continuado, sino que se trata de una suma de intervalos de tiempo; aun así, esta cantidad es muy elevada pues les resta tiempo de hacer otras actividades, como las tareas que les mandan del instituto, compartir momentos con sus familiares y amigos, realizar actividades de ocio y tiempo libre, entre otras. Además, teniendo en cuenta el estudio realizado por la Fundación Pfizer (2009), en el que se encontró que siete de cada diez adolescentes utilizaban internet más de 1,5 horas al día, se puede ver cómo nuestros datos se encuentran relacionados con el mismo, pues un $64,5 \%$ de los adolescentes de nuestro estudio lo utilizan dos o más horas al día.

Por lo que refiere a la frecuencia de problemas debido al uso de las tecnologías, los adolescentes consideran que las tecnologías que mayores problemas les generan son internet y el teléfono móvil. Estos datos se encuentran relacionados con el estudio de Carbonell y cols. (2012), quienes hallaron que un 6,1\% de los adolescentes mostraba problemas frecuentes por el uso de internet (siendo 6,4\% quienes percibían problemas siempre en nuestro estudio) y $2,8 \%$ en el caso del teléfono móvil (siendo 8,5\% en nuestro estudio). Así mismo, parece haber aumentado la consideración de uso problemático del teléfono móvil. Estos datos son importantes, pues representan a una gran cantidad de adolescentes, a la par que llamativos, ya que debido al elevado porcentaje de aquellos que perciben siempre o con frecuencia problemas por el uso de internet y teléfono móvil, podríamos decir que algunos adolescentes sufren una gran interferencia en otras actividades, lo cual Griffiths (2005) define como conflicto y es uno de los componentes de la adicción comportamental. Por otra parte, en el caso de los videojuegos, comparando nuestros resultados con estudios anteriores como el de Protégeles del 2005, en el 
que un $14 \%$ reconoció tener conductas problemáticas, podríamos concluir que ha disminuido el uso problemático en los adolescentes de nuestro estudio, pues solo un 2,1\% lo consideran siempre problemático.

Teniendo en cuenta la relación entre la frecuencia de uso de las TIC y la frecuencia de problemas percibidos, esta es positiva y significativa para internet, videojuegos y televisión, pero no lo es para el teléfono móvil; parece ser que aquellos que utilizan más internet, videojuegos y televisión son quienes mayores problemas perciben. Sin embargo, en el caso del teléfono móvil no ocurre así, lo que podría explicarse por el hecho de que es una tecnología que suele llevarse siempre encima y cuyo uso, a pesar de que pueda ser elevado, puede que no sea percibido como interferente en otras actividades, por lo que probablemente no se es tan consciente de los problemas que genera. En el estudio realizado por Labrador y Villadangos (2010) se encontró de la misma forma correlaciones positivas entre el tiempo de uso de las distintas tecnologías y la percepción de problemas, incluyendo el teléfono móvil.

En conclusión, en la actualidad se puede observar un aumento en el uso de las TIC, como se puede apreciar en los resultados encontrados en el presente estudio, principalmente en relación a internet y teléfono móvil frente a estudios previos. Así mismo, son sorprendentes los resultados encontrados acerca de las horas que los adolescentes utilizan al día internet y el teléfono móvil. De la misma manera, se ha encontrado un importante porcentaje de adolescentes que percibe problemas derivados del uso de internet y del teléfono móvil. En cuanto a la televisión, el uso de la misma se produce principalmente de forma diaria o alguna vez a la semana, aunque las horas que dedican a la misma son inferiores en comparación con las que les dedican a internet y al teléfono móvil y también lo son los problemas percibidos. Por otra parte, en el caso de los videojuegos, se ha encontrado que su uso es inferior al del resto de las tecnologías, así como los problemas derivados del mismo.

Por lo tanto, se ha encontrado un uso abusivo en una parte importante de los adolescentes, principalmente en lo que a internet y teléfono móvil se refiere. Sin embargo, existen dificultades para determinar de un modo preciso cuándo una persona es adicta a las TIC ya que todavía no existen criterios específicos para definir la adicción a las mismas, por ello se hacen necesarios estudios futuros que permitan definir las características y los síntomas de este tipo de adicciones.

Cabe señalar que, con toda la información obtenida tras este estudio, especialmente descriptiva, se dio una charla informativa a los padres para comunicarles los resultados obtenidos y darles las principales pautas de prevención recogidas en la literatura.

\section{Referencias bibliográficas}

American Psychiatric Association (2013). Diagnostic and statistical manual of mental disorders (5th ed.). Arlington, VA: American Psychiatric Publishing.

Carbonell, X., Chamarro, A., Griffiths, M., Oberst, U., Cladellas, R. y Talarn A. (2012). Problematic Internet and cell phone use in Spanish teenagers and young students. Anales de Psicología, 28, 789-796.

Casey, B. J., Tottenham, N., Liston, C. y Durston, S. (2005). Imaging the developing brain: What have we learned about cognitive development? Trends in Cognitive Sciences, 9, 104-110.

Chóliz, M., Villanueva, V. y Chóliz, M. C. (2009). Ellas, ellos y su móvil: Uso, abuso (¿y dependencia?) del teléfono móvil en la adolescencia. Revista Española de Drogodependencias, 34, 74-88. 
Echeburúa, E. y Corral, P. (2010). Adicción a las nuevas tecnologías y a las redes sociales en jóvenes: un nuevo reto. Adicciones, 22(2), 91-96.

Fundación Pfizer (2009). La juventud y las redes sociales en Internet. Madrid: Fundación Pfizer.

Griffiths, M. (2005). A "components" model of addiction within a biopsychosocial framework. Journal of Substance Use, 10, 191-197.

Labrador, F. J. y Villadangos, S. (2009). Adicciones a nuevas tecnologías en adolescentes y jóvenes. En E. Echeburúa, F. J. Labrador y E. Becoña (coords.), Adicción a las nuevas tecnologías en adolescentes y jóvenes (pp. 45-75). Madrid: Pirámide.

Labrador, F. J. y Villadangos, S. (2010). Menores y nuevas tecnologías: conductas indicadoras de posible problema de adicción. Psicothema, 22, 180-188.

Liu, T. y Potenza, M. N. (2007). Problematic Internet use: Clinical implications. CNS Spectrums, 12, 453-466.

Martín, J. M., Beltrán, J. A. y Pérez, L. (2003). ¿Cómo aprender con Internet? Madrid: Fundación.

Montero, I. y León, O. G. (2005). Sistema de clasificación del método en los informes de investigación en Psicología. International Journal of Clinical and Health Psychology, 5, $115-127$.

Protégeles (2005). Seguridad infantil y costumbres de los menores en el empleo de la telefonía móvil. Protégeles y Defensor del Menor (en red).

Sánchez-Carbonell, X., Beranuy, M., Castellana, M., Chamarro, A. y Oberst, U. (2008). La adicción a Internet y al móvil: ¿moda o trastorno? Adicciones, 20, 149-160. 